\title{
Asymptotic stability of positive periodic solution for semilinear evolution equations
}

He Yang ${ }^{1 *}$ and Qiang Li

\section{"Correspondence:}

yanghe256@163.com

'Department of Mathematics,

Shanghai Jiao Tong University,

Shanghai, 200240, People's Republic of China

Full list of author information is

available at the end of the article

\begin{abstract}
The aim of this paper is to study the asymptotic stability of positive periodic solution for semilinear evolution equation in an ordered Banach space $E: u^{\prime}(t)+A u(t)=f(t, u(t))$, $t \in \mathbb{R}^{+}=[0,+\infty)$, where $A: D(A) \subset E \rightarrow E$ is a closed linear operator, and $f: \mathbb{R}^{+} \times E \rightarrow E$ is a continuous mapping which is $\omega$-periodic in $t$. Under order conditions on the nonlinearity $f$, the asymptotic stability results of positive $\omega$-periodic mild solution are obtained on $\mathbb{R}^{+}$by using operator semigroup theory and a monotone iterative technique.
\end{abstract}

MSC: 35B10; 35B40

Keywords: positive $\omega$-periodic mild solution; asymptotic stability; monotone iterative technique

\section{Introduction}

The problems concerning periodic solutions of partial differential equations are an important area of investigation in recent years. Especially, the existence of periodic solutions for the evolution equations has been considered by several authors; see [1-14] and the references therein. In [1], Xiang and Ahmad proved an existence result of the periodic solution to the delay evolution equations in Banach spaces under the assumption that the corresponding initial value problem has an a priori estimate. In [2,3], Liu derived periodic solutions from bounded solutions or ultimate bounded solutions for finite or infinite delay evolution equations in Banach spaces. In [4], Liang et al. proved that if the solutions of the corresponding initial value problem are ultimately bounded, then the delay impulsive evolution equation has a periodic solution. In all these works, the key assumption of $a$ priori boundedness of solutions is employed. In [5], Li studied the existence and uniqueness of positive periodic solutions for semilinear evolution equations in ordered Banach spaces by using a monotone iterative technique. In [6], under the spectral separation conditions of a selfadjoint operator, Li studied the existence and uniqueness of periodic solutions for semilinear evolution equations in Hilbert spaces by using the method of fixed point theorems.

Recently, $\mathrm{Li}$ in [7] investigated the existence and asymptotic stability of time $\omega$-periodic solutions for the delay parabolic boundary value problem (DPBVP),

$$
\left\{\begin{array}{l}
\frac{\partial u(x, t)}{\partial t}+A(x, D) u(x, t) \\
\quad=g\left(x, t, u(x, t), u\left(x, t-\tau_{1}\right), \ldots, u\left(x, t-\tau_{n}\right)\right), \quad(x, t) \in \Omega \times \mathbb{R} \\
\left.u\right|_{\partial \Omega}=0
\end{array}\right.
$$

O2014 Yang and Li; licensee Springer. This is an Open Access article distributed under the terms of the Creative Commons Attribution License (http://creativecommons.org/licenses/by/2.0), which permits unrestricted use, distribution, and reproduction in any medium, provided the original work is properly cited. 
where $\Omega \subset \mathbb{R}^{N}$ is a bounded domain with sufficiently smooth boundary $\partial \Omega$,

$$
A(x, D)=-\sum_{i, j=1}^{N} \frac{\partial}{\partial x_{i}}\left(a_{i j}(x) \frac{\partial}{\partial x_{j}}\right)+a_{0}(x)
$$

is a uniformly elliptic differential operator of divergence form in $\bar{\Omega}$ with the coefficients $a_{i j} \in C^{1+\mu}(\bar{\Omega})(i, j=1,2, \ldots, N)$ and $a_{0} \in C^{\mu}(\bar{\Omega})$ for some $\mu \in(0,1)$. That is, $\left[a_{i j}(x)\right]_{N \times N}$ is a positive define symmetric matrix for every $x \in \bar{\Omega}$, and $\tau_{1}, \tau_{2}, \ldots, \tau_{n}$ are positive constants which denote the time delays. Let $a_{0}(x) \geq 0$ on $\bar{\Omega}, g: \bar{\Omega} \times \mathbb{R} \times \mathbb{R}^{n+1} \rightarrow \mathbb{R}$ be a continuous function which is $\omega$-periodic in $t$. Assume we work under the following assumptions:

$\left(\mathrm{A}_{1}\right)$ There exist positive constants $\beta_{0}, \ldots, \beta_{n}$ such that

$$
\left|g\left(x, t, \eta_{0}, \ldots, \eta_{n}\right)-g\left(x, t, \zeta_{0}, \ldots, \zeta_{n}\right)\right| \leq \sum_{i=0}^{n} \beta_{i}\left|\eta_{i}-\zeta_{i}\right|
$$

for any $\left(x, t, \eta_{0}, \ldots, \eta_{n}\right),\left(x, t, \zeta_{0}, \ldots, \zeta_{n}\right) \in \bar{\Omega} \times \mathbb{R} \times \mathbb{R}^{n+1}$.

$\left(\mathrm{A}_{2}\right) \sum_{i=0}^{n} e^{\lambda_{1} \tau_{i}} \beta_{i}<\lambda_{1}$.

The authors obtained the existence and asymptotic stability of time $\omega$-periodic solutions for the DPBVP (1).

If we have the case without delays, in $\mathbb{R}^{+}$, the DPBVP (1) degenerates into the following problem:

$$
\left\{\begin{array}{l}
\frac{\partial u(x, t)}{\partial t}+A(x, D) u(x, t)=g(x, t, u(x, t)), \quad(x, t) \in \Omega \times \mathbb{R}^{+}, \\
\left.u\right|_{\partial \Omega}=0 .
\end{array}\right.
$$

In this case, the assumptions $\left(\mathrm{A}_{1}\right)$ and $\left(\mathrm{A}_{2}\right)$ degenerate into the following.

$\left(\mathrm{A}_{3}\right)$ There exists a constant $\beta \in\left(0, \lambda_{1}\right)$ such that

$$
\begin{gathered}
|g(x, t, \eta)-g(x, t, \zeta)| \leq \beta|\eta-\zeta|, \\
\text { for any }(x, t, \eta),(x, t, \zeta) \in \bar{\Omega} \times \mathbb{R}^{+} \times \mathbb{R} \text {. }
\end{gathered}
$$

Sometimes the condition $\left(A_{3}\right)$ is not easy to verify in applications. To make the work better applicable, in this paper, we obtain the following result.

Theorem A Let $g(x, t, 0) \geq 0$ and $g(x, t, 0) \not \equiv 0$. Assume that the following conditions are satisfied.

$\left(C_{1}\right)$ For any $R \geq 0$, there exists a constant $M=M(R)>0$ such that

$$
g\left(x, t, \xi_{2}\right)-g\left(x, t, \xi_{1}\right) \geq-M\left(\xi_{2}-\xi_{1}\right), \quad t \in \mathbb{R}^{+},
$$

for $\xi_{1}, \xi_{2} \in \mathbb{R}$ with $0 \leq \xi_{1} \leq \xi_{2},\left|\xi_{i}\right| \leq R(i=1,2)$.

$\left(C_{2}\right)$ There exists a constant $L<\lambda_{1}$ such that

$$
g\left(x, t, \xi_{2}\right)-g\left(x, t, \xi_{1}\right) \leq L\left(\xi_{2}-\xi_{1}\right), \quad t \in \mathbb{R}^{+},
$$

for $\xi_{1}, \xi_{2} \in \mathbb{R}$ with $0 \leq \xi_{1} \leq \xi_{2}$ 
Then the problem (2) has a unique positive time $\omega$-periodic solution and it exponentially attracts every solution of the corresponding initial value problem in $L^{2}(\Omega)$.

Our discussion will be made in the framework of ordered Banach spaces. Let $E$ be an ordered Banach space with norm $\|\cdot\|$, whose positive cone $K$ is normal with normal constant $N=1, A: D(A) \subset E \rightarrow E$ be a closed linear operator, $-A$ generate a $C_{0}$-semigroup $T(t)(t \geq 0)$ in $E$, and let $f: \mathbb{R}^{+} \times E \rightarrow E$ be a continuous mapping which is $\omega$-periodic in $t$. It is well known (see [15]) that for a $C_{0}$-semigroup $T(t)(t \geq 0)$ that there exist $C>0$ and $\delta \in \mathbb{R}$ such that

$$
\|T(t)\| \leq C e^{\delta t}, \quad t \geq 0
$$

Let $v_{0}=\inf \left\{\delta \in R\right.$, there exists $C>0$ such that $\left.\|T(t)\| \leq C e^{\delta t}, t \geq 0\right\}$. Then $v_{0}$ is called the growth exponent of the semigroup $T(t)(t \geq 0)$. Furthermore, $v_{0}$ can also be obtained by the following formula:

$$
v_{0}=\limsup _{t \rightarrow+\infty} \frac{\ln \|T(t)\|}{t}
$$

More generally, we consider the existence and asymptotic stability of time $\omega$-periodic solution for the abstract evolution equation in $E$

$$
u^{\prime}(t)+A u(t)=f(t, u(t)), \quad t \in \mathbb{R}^{+} .
$$

For the abstract evolution equation (3), we obtain the following results.

Theorem 1 Let $E$ be an ordered Banach space, whose positive cone $K$ is normal. Assume that $-A$ generates a positive $C_{0}$-semigroup $T(t)(t \geq 0)$ in $E, f: \mathbb{R}^{+} \times E \rightarrow E$ is a continuous mapping which is $\omega$-periodic in $t$, and $f(t, \theta) \geq \theta, f(t, \theta) \not \equiv \theta$ for $t \in R^{+}$, where $\theta$ is the zero element in E. Assume $f(t, u)$ satisfies the following conditions.

$\left(\mathrm{H}_{1}\right)$ For any $R \geq 0$, there exists a constant $M=M(R)>0$ such that

$$
f(t, y)-f(t, x) \geq-M(y-x), \quad t \in \mathbb{R}^{+},
$$

for $x, y \in E$ with $\theta \leq x \leq y,\|x\| \leq R,\|y\| \leq R$.

$\left(\mathrm{H}_{2}\right)$ There exists a constant $L<-v_{0}$ such that

$$
\begin{aligned}
f(t, y)-f(t, x) & \leq L(y-x), \quad t \in \mathbb{R}^{+}, \\
\text {for } x, y \in E \text { with } \theta & \leq x \leq y .
\end{aligned}
$$

Then the positive $\omega$-periodic mild solution of Eq. (3) is globally asymptotically stable.

If $C_{0}$-semigroup $T(t)$ is continuous in uniform operator topology for every $t>0$ in $E$, it is well known (see [16]) that $\nu_{0}$ can also be determined by $\sigma(A)$ and

$$
\nu_{0}=-\inf \{\operatorname{Re} \lambda: \lambda \in \sigma(A)\}
$$


where $\sigma(A)$ is the spectrum of $A$. We know (see [15]) that compact semigroup is continuous in uniform operator topology for $t>0$. Let $K$ be a regeneration cone, $T(t)(t \geq 0)$ be a compact and positive $C_{0}$-semigroup. By the characteristic of positive semigroups (see [17]) and the Krein-Rutmann theorem, $A$ has the first eigenvalue $\lambda_{1}>0$ and

$$
\lambda_{1}=\inf \{\operatorname{Re} \lambda: \lambda \in \sigma(A)\} .
$$

That is, $\nu_{0}=-\lambda_{1}$. Hence by Theorem 1 , we have the following.

Corollary 1 Let $E$ be an ordered Banach space, whose positive cone $K$ is a normal regeneration cone. Assume that $-A$ generates a compact and positive $C_{0}$-semigroup $T(t)(t \geq 0)$ in $E, f: \mathbb{R}^{+} \times E \rightarrow E$ is a continuous mapping which is w-periodic in $t$ and $f(t, \theta) \geq \theta$, $f(t, \theta) \not \equiv \theta$ for $t \in \mathbb{R}^{+}$. If $f(t, u)$ satisfies the assumptions $\left(\mathrm{H}_{1}\right)$ and

$\left(\mathrm{H}_{2}\right)^{*}$ there exists a constant $L<\lambda_{1}$ such that

$$
\begin{aligned}
f(t, y)-f(t, x) & \leq L(y-x), \quad t \in \mathbb{R}^{+}, \\
\text {for } x, y \in E \text { with } \theta & \leq x \leq y .
\end{aligned}
$$

Then the positive $\omega$-periodic mild solution of Eq. (3) is globally asymptotically stable.

Remark 1 Under the assumptions of Theorem 1 or Corollary 1 , the existence and uniqueness of positive $\omega$-periodic mild solutions for Eq. (3) were obtained by Li in [5]. So, in this paper, we mainly focus on the asymptotic stability of the positive $\omega$-periodic mild solutions.

We apply the above abstract results to the problem (2). Let $E=L^{2}(\Omega), K=\left\{u \in L^{2}(\Omega)\right.$ : $u(x) \geq 0$, a.e. $x \in \Omega\}$. Then $K$ is a normal regeneration cone in $E$. Define an operator $A$ : $D(A) \subset E \rightarrow E$ by

$$
D(A)=H^{2}(\Omega) \cap H_{0}^{1}(\Omega), \quad A u=A(x, D) u .
$$

It is well known (see [7]) that $-A$ generates a compact $C_{0}$-semigroup in $E$ which is also positive. Define a mapping $f: \mathbb{R}^{+} \times E \rightarrow E$ by

$$
f(t, u)=g(\cdot, t, u(\cdot)), \quad u \in L^{2}(\Omega), t \in \mathbb{R}^{+} .
$$

It is clear that $f: \mathbb{R}^{+} \times E \rightarrow E$ is continuous and it is $\omega$-periodic in $t$. Thus, the problem (2) is rewritten into the form of abstract evolution equation (3). When the conditions $\left(C_{1}\right)$ and $\left(\mathrm{C}_{2}\right)$ of Theorem A are satisfied, the mapping $f: \mathbb{R}^{+} \times E \rightarrow E$ defined by (4) satisfies the conditions $\left(\mathrm{H}_{1}\right)$ and $\left(\mathrm{H}_{2}\right)^{*}$. Hence, by Corollary 1, we obtain the conclusion of Theorem A.

The abstract result of Theorem 1 will be proved in Section 3. In Section 2, some preliminary conclusions are given.

\section{Preliminaries}

Let $E$ be an ordered Banach space, whose positive cone $K$ is normal, $A: D(A) \rightarrow E$ be a closed linear operator in $E$. Denote by $C([0, \omega], E)$ the continuous function space from 
$[0, \omega]$ to $E$. Let $C_{\omega}\left(\mathbb{R}^{+}, E\right)$ be the Banach space $\left\{u \in C\left(\mathbb{R}^{+}, E\right): u(t)=u(t+\omega), t \in \mathbb{R}^{+}\right\}$endowed with the maximum norm $\|u\|_{C}=\max _{t \in[0, \omega]}\|u(t)\|$. We first consider the existence of the initial value problem (IVP) of the evolution equation in $E$

$$
\left\{\begin{array}{l}
u^{\prime}(t)+A u(t)=f(t, u(t)), \quad t>0, \\
u(0)=x_{0} .
\end{array}\right.
$$

For IVP (5), we obtain the following existence result.

Lemma 1 Let $E$ be an ordered Banach space, whose positive cone $K$ is normal. Assume that - A generates a positive $C_{0}$-semigroup $T(t)(t \geq 0)$ in $E, f: \mathbb{R}^{+} \times K \rightarrow E$ is continuous and $f(t, \theta) \geq \theta, f(t, \theta) \not \equiv \theta$ for $t \in \mathbb{R}^{+}$. If $x_{0} \in K$ and $f(t, u)$ satisfies the conditions $\left(\mathrm{H}_{1}\right)$ and $\left(\mathrm{H}_{2}\right)$, then IVP (5) has a unique mild solution.

Proof Let $h_{0}(t)=f(t, \theta)$. Then $h_{0} \in C\left(\mathbb{R}^{+}, E\right), h_{0}(t) \geq \theta$ and $h_{0}(t) \not \equiv \theta$. We first consider the initial value problem of linear evolution equation (LIVP)

$$
\left\{\begin{array}{l}
u^{\prime}(t)+(A-L I) u(t)=h_{0}(t), \quad t>0 \\
u(0)=x_{0}
\end{array}\right.
$$

It is well known (see [15]) that the LIVP (6) has a unique mild solution $u_{0} \in C\left(\mathbb{R}^{+}, E\right)$ expressed by

$$
u_{0}(t)=S_{1}(t) x_{0}+\int_{0}^{t} S_{1}(t-s) h_{0}(s) d s, \quad t \geq 0
$$

where $S_{1}(t)=e^{L t} T(t)(t \geq 0)$ is a positive $C_{0}$-semigroup generated by $-(A-L I)$, whose norm satisfies $\left\|S_{1}(t)\right\| \leq C e^{\left(L+v_{0}\right) t} \leq C$ for $t \geq 0$. Hence, we have

$$
\left\|u_{0}(t)\right\| \leq C\left\|x_{0}\right\|+C \int_{0}^{t} e^{\left(L+\nu_{0}\right)(t-s)} d s \cdot\left\|h_{0}\right\|_{C} \leq C\left\|x_{0}\right\|+\frac{C\left\|h_{0}\right\|_{C}}{-\left(L+v_{0}\right)} \triangleq \bar{R} .
$$

Let $v_{0} \equiv \theta, w_{0}=u_{0}$. By $h_{0}(t) \geq \theta, h_{0}(t) \not \equiv \theta, x_{0} \in K$ and the positive property of semigroup $S_{1}(t)(t \geq 0)$, we see that $v_{0} \leq w_{0}$. Let $M=M(\bar{R})>0$ be the constant in assumption $\left(\mathrm{H}_{1}\right)$. We consider the following IVP of the evolution equation:

$$
\left\{\begin{array}{l}
u^{\prime}(t)+(A+M I) u(t)=f(t, u(t))+M u(t), \quad t>0 \\
u(0)=x_{0}
\end{array}\right.
$$

Without loss of generality, we assume $M>-L$ (otherwise, replacing $M$ by $M+|L|$, the assumption $\left(\mathrm{H}_{1}\right)$ still holds). Then the operator $-(A+M I)$ generates a positive $C_{0}$-semigroup $S_{2}(t)=e^{-M t} T(t)(t \geq 0)$, whose norm satisfies $\left\|S_{2}(t)\right\| \leq C e^{-\left(M-\nu_{0}\right) t} \leq C$ for $t \geq 0$.

Define the operator $Q$ by

$$
(Q u)(t)=S_{2}(t) x_{0}+\int_{0}^{t} S_{2}(t-s)[f(s, u(s))+M u(s)] d s, \quad t \geq 0 .
$$

It is clear that the mild solution of IVP (7) is equivalent to the fixed point of operator $Q$. 
Let $D=\left[v_{0}, w_{0}\right]$. It follows from assumption $\left(\mathrm{H}_{1}\right)$ that $Q: D \rightarrow C\left(\mathbb{R}^{+}, E\right)$ is a continuously increasing operator. Let

$$
v_{n}=Q v_{n-1}, \quad w_{n}=Q w_{n-1}, \quad n=1,2, \ldots
$$

Then

$$
\theta=v_{0} \leq v_{1} \leq \cdots \leq v_{n} \leq \cdots \leq w_{n} \leq \cdots \leq w_{1} \leq w_{0} .
$$

Therefore, for any $t \geq 0$, we have

$$
\begin{aligned}
\theta & \leq w_{n}(t)-v_{n}(t) \\
& =\int_{0}^{t} S_{2}(t-s)\left[f\left(s, w_{n-1}(s)\right)-f\left(s, v_{n-1}(s)\right)+M\left(w_{n-1}(s)-v_{n-1}(s)\right)\right] d s \\
& \leq(M+L) \int_{0}^{t} S_{2}(t-s)\left(w_{n-1}(s)-v_{n-1}(s)\right) d s .
\end{aligned}
$$

By the normality of cone $K$ in $E$, we have

$$
\left\|w_{n}(t)-v_{n}(t)\right\| \leq C(M+L) \int_{0}^{t}\left\|w_{n-1}(s)-v_{n-1}(s)\right\| d s
$$

Continuing such a procedure, we have

$$
\left\|w_{n}(t)-v_{n}(t)\right\| \leq \frac{[C(M+L)]^{n}}{n !} t^{n} \cdot\left\|w_{0}\right\|_{C} \rightarrow 0 \quad(n \rightarrow \infty) .
$$

This implies that there is a unique $u^{*} \in C\left(\mathbb{R}^{+}, E\right)$ such that

$$
u^{*}(t)=\lim _{n \rightarrow \infty} w_{n}(t)=\lim _{n \rightarrow \infty} v_{n}(t), \quad t \geq 0 .
$$

Combining this with (9), since the convergence is uniform in each compact interval and the operator $Q$ is continuous, we obtain $u^{*}=Q u^{*}$. Therefore, $u\left(t ; x_{0}\right):=u^{*}(t)$ is the unique mild solution of IVP (7) on $\mathbb{R}^{+}$. This proof is completed.

To prove our main result, we also need the following lemma.

Lemma 2 Let $y_{1}, y_{2} \in E$ with $\theta \leq y_{1} \leq y_{2}$. Then $u\left(t ; y_{1}\right) \leq u\left(t ; y_{2}\right)$.

Proof Consider the following two initial value problems:

$$
\left\{\begin{array}{l}
u^{\prime}(t)+A u(t)=f(t, u(t)), \quad t>0 \\
u(0)=y_{1}
\end{array}\right.
$$

and

$$
\left\{\begin{array}{l}
u^{\prime}(t)+A u(t)=f(t, u(t)), \quad t>0 \\
u(0)=y_{2}
\end{array}\right.
$$


Let $x_{0}=y_{1}+y_{2}$. Then the solution of LIVP (6) is the $w_{0}$ corresponding to IVP (11) and IVP (12). Let $v_{0}=\theta$. A similar argument as in Lemma 1 shows that

$$
v_{i, n}=Q v_{i, n-1}, \quad w_{i, n}=Q w_{i, n-1}, \quad i=1,2, n=1,2, \ldots
$$

and

$$
u\left(t ; y_{1}\right)=\lim _{n \rightarrow \infty} v_{1, n}(t)=\lim _{n \rightarrow \infty} w_{1, n}(t), \quad u\left(t ; y_{2}\right)=\lim _{n \rightarrow \infty} v_{2, n}(t)=\lim _{n \rightarrow \infty} w_{2, n}(t), \quad t \geq 0
$$

For any $t \geq 0$, noticing that $v_{1,0}(t)=v_{2,0}(t) \equiv \theta$ and $\theta \leq y_{1} \leq y_{2}$, it follows from the positivity of the operators $S_{2}(t)$ and

$$
\begin{aligned}
& v_{1,1}(t)=\left(Q v_{1,0}\right)(t)=S_{2}(t) y_{1}+\int_{0}^{t} S_{2}(t-s) h_{0}(s) d s, \\
& v_{2,1}(t)=\left(Q v_{2,0}\right)(t)=S_{2}(t) y_{2}+\int_{0}^{t} S_{2}(t-s) h_{0}(s) d s
\end{aligned}
$$

that $v_{1,1}(t) \leq v_{2,1}(t)$. Inductively, when $v_{1, n-1}(t) \leq v_{2, n-1}(t)$, it follows from

$$
\begin{aligned}
& v_{1, n}(t)=\left(Q v_{1, n-1}\right)(t)=S_{2}(t) y_{1}+\int_{0}^{t} S_{2}(t-s)\left[f\left(s, v_{1, n-1}(s)\right)+M v_{1, n-1}(s)\right] d s, \\
& v_{2, n}(t)=\left(Q v_{2, n-1}\right)(t)=S_{2}(t) y_{2}+\int_{0}^{t} S_{2}(t-s)\left[f\left(s, v_{2, n-1}(s)\right)+M v_{2, n-1}(s)\right] d s
\end{aligned}
$$

that

$$
v_{1, n}(t) \leq v_{2, n}(t), \quad t \geq 0 .
$$

Taking the limits on both sides of inequality (13) as $n \rightarrow \infty$, we obtain

$$
u\left(t ; y_{1}\right) \leq u\left(t ; y_{2}\right)
$$

This proof is completed.

For the existence and uniqueness of $\omega$-periodic mild solutions of Eq. (3), we have the following result.

Lemma 3 (see [5]) Let $E$ be an ordered Banach space, whose positive cone $K$ is normal. Assume that $-A$ generates a positive $C_{0}$-semigroup $T(t)(t \geq 0)$ in $E, f: \mathbb{R}^{+} \times K \rightarrow E$ is a continuous mapping which is $\omega$-periodic in $t$ and $f(t, \theta) \geq \theta, f(t, \theta) \not \equiv \theta$ for $t \geq 0$. If $f(t, u)$ satisfies the conditions $\left(\mathrm{H}_{1}\right)$ and $\left(\mathrm{H}_{2}\right)$, then Eq. (3) has a unique positive $\omega$-periodic mild solution on $\mathbb{R}^{+}$.

\section{The proof of Theorem 1}

Proof of Theorem 1 Define an equivalent norm $|\cdot|_{E}$ in $E$ by

$$
|x|_{E}=\sup _{t \geq 0}\left\|e^{\left(M-\nu_{0}\right) t} S_{2}(t) x\right\|
$$


Then $\|x\| \leq|x|_{E} \leq C\|x\|$ and

$$
\begin{aligned}
\left|S_{2}(t) x\right|_{E} & =\sup _{s \geq 0}\left\|e^{\left(M-v_{0}\right) s} S_{2}(s) \cdot S_{2}(t) x\right\|=\sup _{s \geq 0}\left\|e^{\left(M-v_{0}\right) s} S_{2}(t+s) x\right\| \\
& =e^{-\left(M-v_{0}\right) t} \cdot \sup _{s \geq 0}\left\|e^{\left(M-v_{0}\right)(t+s)} S_{2}(t+s) x\right\| \\
& \leq e^{-\left(M-v_{0}\right) t} \cdot|x|_{E}
\end{aligned}
$$

which implies that $\left|S_{2}(t)\right|_{E} \leq e^{-\left(M-v_{0}\right) t}$.

From Lemma 3, Eq. (3) has a unique positive $\omega$-periodic mild solution $\tilde{u}$ on $\mathbb{R}^{+}$. By Lemma 1, IVP (5) has a unique positive mild solution $u=u\left(t ; x_{0}\right) \in C\left(\mathbb{R}^{+}, K\right)$. Let $y_{0}=\widetilde{u}(0)$. Then $\tilde{u}(t)=u\left(t ; y_{0}\right)$. Setting $x_{1}:=y_{0}+x_{0}$, then $x_{1} \geq y_{0}, x_{1} \geq x_{0}$. By Lemma 2 , we see that $\theta \leq \widetilde{u}(t) \leq u\left(t ; x_{1}\right), \theta \leq u\left(t ; x_{0}\right) \leq u\left(t ; x_{1}\right)$. Setting $u_{1}(t)=u\left(t ; x_{1}\right), u^{*}(t)=u\left(t ; x_{0}\right)$, by the semigroup representation of the solutions, we have

$$
\begin{aligned}
\theta \leq & u_{1}(t)-\widetilde{u}(t)=\left(Q u_{1}\right)(t)-(Q \widetilde{u})(t) \\
= & S_{2}(t) x_{1}+\int_{0}^{t} S_{2}(t-s)\left(f\left(s, u_{1}(s)\right)+M u_{1}(s)\right) d s \\
& -S_{2}(t) y_{0}-\int_{0}^{t} S_{2}(t-s)(f(s, \widetilde{u}(s))+M \widetilde{u}(s)) d s \\
\leq & S_{2}(t)\left(x_{1}-y_{0}\right)+\int_{0}^{t} S_{2}(t-s)\left[f\left(s, u_{1}(s)\right)-f(s, \widetilde{u}(s))+M\left(u_{1}(s)-\widetilde{u}(s)\right)\right] d s \\
\leq & S_{2}(t)\left(x_{1}-y_{0}\right)+(M+L) \int_{0}^{t} S_{2}(t-s)\left(u_{1}(s)-\widetilde{u}(s)\right) d s .
\end{aligned}
$$

By the normality of cone $K$ in $E$, we have

$$
\begin{aligned}
\left|u_{1}(t)-\widetilde{u}(t)\right|_{E} & \leq\left|S_{2}(t)\left(x_{1}-y_{0}\right)\right|_{E}+(M+L)\left|\int_{0}^{t} S_{2}(t-s)\left(u_{1}(s)-\widetilde{u}(s)\right) d s\right|_{E} \\
& \leq e^{-\left(M-v_{0}\right) t}\left|x_{1}-y_{0}\right|_{E}+(M+L) \int_{0}^{t} e^{-\left(M-v_{0}\right)(t-s)}\left|u_{1}(s)-\widetilde{u}(s)\right|_{E} d s,
\end{aligned}
$$

that is,

$$
e^{\left(M-v_{0}\right) t}\left|u_{1}(t)-\widetilde{u}(t)\right|_{E} \leq\left|x_{1}-y_{0}\right|_{E}+(M+L) \int_{0}^{t} e^{\left(M-v_{0}\right) s}\left|u_{1}(s)-\widetilde{u}(s)\right|_{E} d s .
$$

By the Gronwall-Bellman inequality, we have

$$
\left|u_{1}(t)-\widetilde{u}(t)\right|_{E} \leq\left|x_{1}-y_{0}\right|_{E} \cdot e^{\left(L+\nu_{0}\right) t} \rightarrow 0 \quad(t \rightarrow \infty) .
$$

Similarly, $\left|u_{1}(t)-u^{*}(t)\right|_{E} \rightarrow 0(t \rightarrow \infty)$. Therefore, we obtain

$$
\left|\widetilde{u}(t)-u^{*}(t)\right|_{E} \leq\left|\widetilde{u}(t)-u_{1}(t)\right|_{E}+\left|u_{1}(t)-u^{*}(t)\right|_{E} \rightarrow 0 \quad(t \rightarrow \infty)
$$

This proof is completed. 


\section{Application}

To illustrate our results, we consider the semilinear partial differential equations in $\mathbb{R}^{2}$ of the form

$$
\left\{\begin{array}{l}
\frac{\partial u(x, t)}{\partial t}+\frac{\partial u(x, t)}{\partial x}=g(x, t, u(x, t)), \quad(x, t) \in \mathbb{R}^{2}, \\
u(x+2 \pi, t)=u(x, t+2 \pi)=u(x, t), \quad(x, t) \in \mathbb{R}^{2},
\end{array}\right.
$$

where $g \in C^{1}\left(\mathbb{R}^{3}\right)$ is $2 \pi$-periodic both in $x$ and $t$.

Let $E=C_{2 \pi}(\mathbb{R})$. Define an operator $A$ in $E$ by

$$
D(A)=C_{2 \pi}^{1}(\mathbb{R}), \quad A u=\frac{\partial u(x, t)}{\partial x} .
$$

By [5], $-A$ generates a contraction $C_{0}$-semigroup $T(t)(t \geq 0)$ in $E$, which is also a positive $C_{0}$-semigroup. By the contraction property of $T(t)(t \geq 0)$, we know that $v_{0} \leq 0$.

Let $f(t, u(t))(\cdot)=g(\cdot, t, u(\cdot, t))$. Then $f: \mathbb{R} \times E \rightarrow E$ is continuous and is $2 \pi$-periodic in $t$. From Theorem 1, we can obtain the following.

Theorem 2 Let $g \in C^{1}\left(\mathbb{R}^{3}\right)$ which is $2 \pi$-periodic both in $x$ and $t$, and $g(x, t, 0) \geq 0$, $g(x, t, 0) \not \equiv 0$. Assume that the following conditions are satisfied:

$\left(\mathrm{P}_{1}\right)$ For any $R \geq 0$, there exists a constant $M=M(R)>0$ such that

$$
g\left(x, t, \xi_{2}\right)-g\left(x, t, \xi_{1}\right) \geq-M\left(\xi_{2}-\xi_{1}\right), \quad t \in \mathbb{R}^{+},
$$

for $\xi_{1}, \xi_{2} \in \mathbb{R}$ with $0 \leq \xi_{1} \leq \xi_{2},\left|\xi_{i}\right| \leq R(i=1,2)$

$\left(\mathrm{P}_{2}\right)$ There exists a constant $L<-v_{0}$ such that

$$
g\left(x, t, \xi_{2}\right)-g\left(x, t, \xi_{1}\right) \leq L\left(\xi_{2}-\xi_{1}\right), \quad t \in \mathbb{R}^{+},
$$

for $\xi_{1}, \xi_{2} \in \mathbb{R}$ with $0 \leq \xi_{1} \leq \xi_{2}$

Then the problem (14) has a unique double $2 \pi$-periodic mild solution in $C_{2 \pi}(\mathbb{R}, E)$ which is globally asymptotic stable.

Remark 2 It is clear that if $\sup g_{u}(x, t, u)<0$, then the assumptions $\left(\mathrm{P}_{1}\right)$ and $\left(\mathrm{P}_{2}\right)$ hold automatically.

\footnotetext{
Competing interests

The authors declare that they have no competing interests.
}

\section{Authors' contributions}

All authors contributed equally to the writing of this paper. All authors read and approved the final manuscript.

\section{Author details}

'Department of Mathematics, Shanghai Jiao Tong University, Shanghai, 200240, People's Republic of China. ${ }^{2}$ Department of Mathematics, Northwest Normal University, Lanzhou, 730070, People's Republic of China.

\section{Acknowledgements}

The authors are grateful to the referees for their helpful comments and suggestions. Research supported by NNSF of China (No. 11261053), NSF of Gansu Province (No. 1308RJZA217) and Project of NWNU-LKQN-11-3. 


\section{References}

1. Xiang, X, Ahmed, N: Existence of periodic solutions of semilinear evolution equations with time lags. Nonlinear Anal. 18, 1063-1070 (1992)

2. Liu, J: Bounded and periodic solutions of finite delays evolution equations. Nonlinear Anal. 34, 101-111 (1998)

3. Liu, J: Bounded and periodic solutions of infinite delay evolution equations. J. Math. Anal. Appl. 286, 705-712 (2003)

4. Liang, J, Liu, J, Xiao, T: Periodic solutions of delay impulsive differential equations. Nonlinear Anal. 74, 6835-6842 (2011)

5. Li, Y: Existence and uniqueness of positive periodic solutions for abstract semilinear evolution equations. J. Syst. Sci. Math. Sci. 25, 720-728 (2005) (in Chinese)

6. Li, Y: Existence and uniqueness of periodic solution for a class of semilinear evolution equations. J. Math. Anal. Appl. 349, 226-234 (2009)

7. Li, Y: Existence and asymptotic stability of periodic solution for evolution equations with delays. J. Funct. Anal. 261, 1309-1324 (2011)

8. Amann, H: Periodic solutions of semilinear parabolic equations. In: Cesari, L, Kannan, R, Weinberger, R (eds.) Nonlinear Analysis: A Collection of Papers in Honor of Erich H. Rothe, pp. 1-29. Academic Press, New York (1978)

9. Lizama, C: Fourier multiplier and periodic solutions of delay equations in Banach spaces. J. Math. Anal. Appl. 324(2), 921-933 (2006)

10. Cuevas, C, Lizama, C, Soto, H: Asymptotic periodicity for strongly damped wave equations. Abstr. Appl. Anal. 2013, Article ID 308616 (2013)

11. Agarwal, R, Cuevas, C, Soto, H, El-Gebeily, M: Asymptotic periodicity for some evolution equations in Banach spaces. Nonlinear Anal. 74(5), 1769-1798 (2011)

12. Agarwal, R, Cuevas, C, Soto, H: Pseudo-almost periodic solutions of a class of semilinear fractional differential equations. J. Appl. Math. Comput. 37(1), 625-634 (2011)

13. Cuevas, C, Sepúlveda, A, Soto, H: Almost periodic and pseudo-almost periodic solutions to fractional differential and integro-differential equations. Appl. Math. Comput. 218, 1735-1745 (2011)

14. Cuevas, C, Pierri, M, Sepúlveda, A: Weighted S-asymptotically $\omega$-periodic solutions of a class of fractional differential equations. Adv. Differ. Equ. 2011, Article ID 584874 (2011)

15. Pazy, A: Semigroups of Linear Operators and Applications to Partial Differential Equations. Springer, Berlin (1983)

16. Triggiani, R: On the stabilizability problem in Banach spaces. J. Math. Anal. Appl. 52, 383-403 (1975)

17. Li, Y: Positive solution for abstract semilinear evolution equations and its applications. Acta Math. Sin. 39, 666-672 (1996) (in Chinese)

10.1186/1687-1847-2014-197

Cite this article as: Yang and Li: Asymptotic stability of positive periodic solution for semilinear evolution equations. Advances in Difference Equations 2014, 2014:197

\section{Submit your manuscript to a SpringerOpen ${ }^{\ominus}$ journal and benefit from:}

- Convenient online submission

- Rigorous peer review

- Immediate publication on acceptance

Open access: articles freely available online

- High visibility within the field

- Retaining the copyright to your article 